\title{
P53 and MDM2 Over-expression and Five-year Survival of Kidney Cancer Patients Undergoing Radical Nephrectomy - Iranian Experience
}

\author{
Maryam Abolhasani ${ }^{1,2 *}$, Sareh Salarinejad ${ }^{3}$, Mojgan Asgari ${ }^{1,2}$
}

\begin{abstract}
Background: Relatively little is known with certainty about the status and role of p53 or MDM2 in predicting prognosis and survival of renal cell carcinoma. The present study aimed to determine the value of P53 and MDM2 over-expression, alone and simultaneously, to predict five-year survival of patients with kidney cancer in Iran. Materials and Methods: Patients with kidney cancer referred to Hasheminejad Kidney Center between 2007 and 2009, underwent radical nephrectomy and had pathology reports of clear cell, papillary or chromophobe renal cell carcinoma were included in our cohort study. Other histological types of renal cell carcinoma were not included. The patients with missed, incomplete or poor quality paraffin blocks were also excluded. Overall ninety one patients met the inclusion and exclusion criteria. To assess the histopathological features of the tumor, immunohistochemical (IHC) staining of formalin fixed, paraffin-embedded tumor samples were performed. The five-year survival was determined by the patients' medical files and telephone following-up. Results: In total, 1.1\% of all samples were revealed to be positive for P53. Also, $20.8 \%$ of all samples were revealed to be positive for MDM2.The patients were all followed for 5 years. In this regard, 5-year mortality was 30.5\% and thus 5-year survival was $\mathbf{8 5 . 3 \%}$. According to the Cox proportional hazard analysis, positive P53 marker was only predictor for patients' 5 -year survival that the presence of positive p53 increased the risk for long-term mortality up to 2.8 times $(\mathrm{HR}=2.798,95 \% \mathrm{CI}$ : 1.176-6.660, $\mathrm{P}=\mathbf{0 . 0 2 0})$. However, the presence of MDM2 could not predict long-term mortality. In this regard, analysis by the ROC curve showed a limited role for predicting long-term survival by confirming $\mathrm{P53}$ positivity (AUC=0.610, 95\% CI: 0.471-.750, $\mathrm{P}=0.106)$. The best cutoff point for P53 to predict mortality was 0.5 yielding a low sensitivity $(32.0 \%)$ but a high specificity $(97.9 \%)$. In similar analysis, measurement of MDM2 positivity could not predict mortality (AUC=0.449,95\% CI: 0.316-.583, $P=0.455)$. Conclusions: The simultaneous presence of both $P 53$ and MDM2 markers in our population is a rare phenomenon and the presence of these markers may not predict long-term survival in patients who undergoing radical nephrectomy.
\end{abstract}

Keywords: Renal cell carcinoma - p53 - MDM2 - 5-year survival - mortality

Asian Pac J Cancer Prev, 16 (12), 5043-5047

\section{Introduction}

More than two thousands of people around the world suffer from different types of kidney carcinoma with the highest prevalence in North America and Europe and the lowest in central Africa. Kidney cancer is the third common urologic cancer in Iran following bladder and prostate cancers (Akbari et al., 2005; Basiri et al., 2014). Also, a quarter of the affected patients face locally invasive or metastatic cancer (Jemal et al., 2005). In this regard, one-third of individuals with renal carcinoma undergo resection of localized disease may experience a recurrence. Hence, there is a great need for more effective surgical and medical therapies as well as accurate estimating survival of the patients. Besides traditional risk factors for renal cell carcinoma including smoking, central obesity, existing kidney conditions, hepatitis infections, long-term use of non-steroidal anti inflammatory drugs, hypertension, low intake of fruits and vegetables, and family history of kidney cancer (Chow et al., 2000; Chow et al., 2010; Karami et al., 2013; Sanfilippo et al., 2014; Washio et al., 2014), some genetic-based causes and mutations involved in protein over-expression and up-regulation have been recently identified to develop renal cell carcinoma (Moore et al., 2005; Haas et al., 2014). Moreover, the poor prognosis of cancer not only can be predicted by clinical (palpable abdominal mass) (Yap et al., 2013), some paraclinical factors (such as a high level of serum 
lactate dehydrogenase, a low hemoglobin level, and a high corrected level of serum calcium), and histological factors (such as tumor staging, tumor grading, tumor size, degree of invasion and metastasis, histological type, and nuclear grade), but also the variants of gene mutations can be determinants for poor prognosis of the disease (Moch et al., 2013; Shuch et al., 2014).

One of the recent investigated molecules studied that involved in gene mutational pathways for developing renal cell carcinoma is the P53 gene and its related specific protein (Uzunlar et al., 2005). The p53 protein accumulates at times of cellular or genotoxic stress, when it functions primarily as a transcription factor to promote cell cycle arrest and DNA repair, to initiate and maintain a senescent phenotype, or to promote apoptosis if the normal cellular conditions are not restored (Zigeuner et al., 2004). The importance of the tumor suppressor function of p53 is highlighted by the high frequency of tumors that occur in individuals with a monoallelic germline mutation of p53 (Kim et al., 2004). The potentially lethal activities of p53 are regulated by a proto-oncogene named MDM2 (murine double minute 2). Several biological studies could demonstrated that p53 and MDM2 exist in an auto regulatory feedback loop that binding of MDM2 to p53 can block p53 transcriptional activity by preventing it from interacting with the transcriptional machinery (Uchida et al., 2002; Kim et al., 2004). On the other hand, the increased levels of MDM2 can lead to a reduction in p53 levels. With respect to cancer, MDM2 over expression has been associated with increased metastasis and advanced disease.

Recently, the prognostic implications of both p53 and MDM2 expression were more evaluated. It has been shown that the frequency of p53 mutations reported in a wide spectrum between $0 \%$ and $44 \%$ (Olumi et al., 2001). Also, its value to assess prognosis and overall survival of caner is uncertain. It has been also observed that the expression of P53 may be linked to expression of MDM2 and thus higher prognostic value of expressing both molecules is also now hypothesized (Ljungberg et al., 2001). However, relatively little is known with certainty about the status and role of p53 or MDM2 in predicting prognosis and survival of renal cell carcinoma. The present study aimed to determine the value of P53 and MDM2 over-expression, alone and simultaneously, to predict fiveyear survival of patients with kidney cancer.

\section{Materials and Methods}

In a cohort study, patients with kidney cancer referred to Hasheminejad Kidney Center between 2007 and 2009, underwent radical nephrectomy and had pathology reports of clear cell, papillary or chromophobe renal cell carcinoma were included into the study. Other histological types of renal cell carcinoma were excluded from the study. Baseline characteristics regarding demographics, medical history, medications, and pathologic data included pTNM stage, tumor size, Fuhrman nuclear grade and the presence of vascular invasion were collected by reviewing the recorded files. The patients with missed, incomplete or poor quality paraffin blocks were also excluded from the study. Overall ninety one patients met the criteria.

For evaluation of immunohistochemical reactivity, representative areas from each case were selected from paraffin blocks based on hematoxylin and eosin-stained sections. IHC staining was carried out using an anti-p53 monoclonal antibody (NOVOCASTRA clone DO-7, ready to use for immunohistochemistry) and anti-MDM2 antibody (NOVOCASTRA clone 1B10 used at a dilution of 1:200 for immunohistochemistry). Both positive and negative control samples of both P53 and MDM2 parameters were taken in every IHC examination. Breast cancer and well differentiated liposarcoma were used as positive controls and cerebellar tissue and pleomorphic liposarcoma for negative controls of P53 and MDM2 respectively. All IHC slides were examined and scored by two pathologists independently and the mean were assigned. The intensity of nuclear immunostaining of P53 and MDM2 in individual tumor cells was scored on a scale of 0 (no staining), 1 (mild staining), 2 (moderate staining), and 3(strongest intensity), and the percentage of cells staining at each intensity was scored as 1 ( 0 to 2 percent), 2 ( 3 to 10 percent), 3 (11 to 50 percent), and 4 (more than 50 percent). Cases with intensity score of 3 and percentage score of 4 were considered as positive. Other combinations of intensity and percentage scores regarded as negative.

The five-year survival was determined by the patients' medical files and telephone following-up.

For analysis of our experience, results were presented as mean \pm standard deviation (SD) for quantitative variables and were summarized by absolute frequencies and percentages for categorical variables. Continuous variables were compared using t test, quantitative variables were, on the other hand, compared using chi-square test or Fisher's exact test .Cancer-specific survival and overall survival were estimated using the Kaplan-Meier method, Multivariate Cox proportional hazards models using a backward stepwise selection method with the likelihood ratio criterion (inclusion/exclusion:P0.05/P.10) were used to determine the independent influence of variables on survival. All statistical tests were 2-sided and were performed at a significance level of 0.05. All analyses were performed using SPSS version 21.0 (SPSS Inc., Chicago, IL).

\section{Results}

Overall, ninety one patients were assessed. The mean age of patients was $56.7 \pm 12.3$ years (ranged 28 to 86 years) and $58.2 \%$ were male. The mean size of lesions was also $8.5 \pm 5.4 \mathrm{~cm}$ ranged 2.5 to $18 \mathrm{~cm}$. left-sided lesions were observed in $50.5 \%$ and right-sided in $49.5 \%$. Regarding type of lesions, $69.2 \%$ were clear cell, $19.8 \%$ were chromophobe, and $11 \%$ were papillary. $15.1 \%$ were categorized as grade $1,52 \%$ as grade $2,27.0 \%$ as grade 3 , and $6.2 \%$ as grade 4 . With regard to organ involvement, renal sinus was involved in $71.3 \%$, perirenal fat involvement in $23 \%$, renal pelvis involvement in $7 \%$, renal vein involvement in $9 \%$, vena cava involvement in $2.2 \%$ and adrenal gland involvement in $3.2 \%$. Necrosis was noted in $51 \%$ of cases. Also, vascular and neural 
P53 and MDM2 Over-expression and Survival of Patients with Kidney Cancer - an Iranian Experience

invasions were observed in $23 \%$ and $3.3 \%$ respectively. Lymph node involvement was noted in $8 \%$. Recurrence was revealed in $1.1 \%$, while metastasis in 5-7 years follow up was occurred in $17.6 \%$.

Regarding the intensity of immunostaining for anti-p53 monoclonal antibody, the score was 0 in $82.4 \%, 1$ in $7.7 \%$, 2 in $5.5 \%$, and 3 in $4.4 \%$. In this regard, the percentage of cells staining at intensity was scored as 1 in $78.0 \%$, 2 in $17.6 \%, 3$ in $3.3 \%$, and 4 in $1.1 \%$. Thus, $1.1 \%$ of all samples were revealed to be positive for P53. Regarding intensity of immunostaining for anti-MDM2 antibody, the score was 0 in $31.9 \%, 1$ in $17.6 \%, 2$ in $19.8 \%$, and 3 in $30.8 \%$. Also, the percentage of cells staining at intensity was scored as 1 in $33.0 \%, 2$ in $12.1 \%, 3$ in $29.7 \%$, and 4 in $25.3 \%$. Thus, $20.8 \%$ of all samples were revealed to be positive for MDM2. Regarding risk factors, $14.3 \%$ were smoker and $12.1 \%$ were hypertensive. Regarding stage of disease, $18.6 \%$ had stage I, 5.4\% had stage II, $69.2 \%$ had stage III, and $6.5 \%$ had stage IV.

The patients were all followed for 5 years survival. In

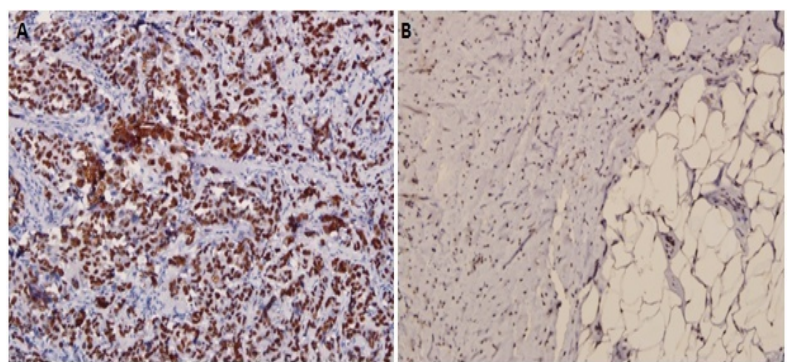

Figure 1. Positive Controls for p53 and MDM2 (A) p53 Nuclear Positivity in Invasive Ductal Carcinoma of Breast Cancer with Original Magnification $\times 20$, (B) MDM2 Nuclear Positivity in Well Differentiated Liposarcoma with Original Magnification $\times 20$

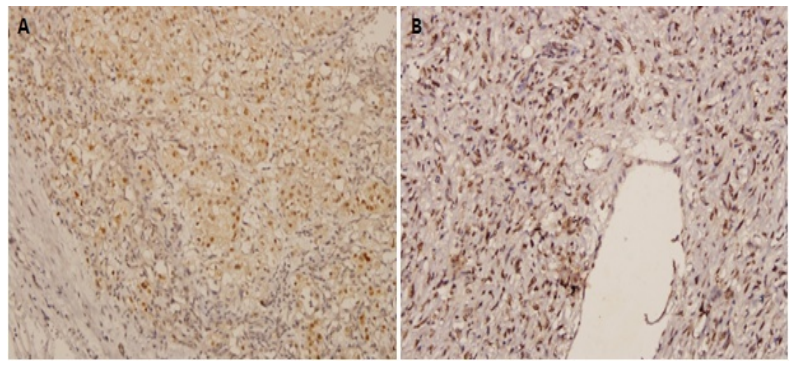

Figure 2. Positive Nuclear Staining of p53 and MDM2 in Renal Cell Carcinoma. (A) p53 Positive Immunoreaction with Original Magnification $\times 20$, (B) MDM2 Positive Immunoreaction with Original Magnification $\times 20$

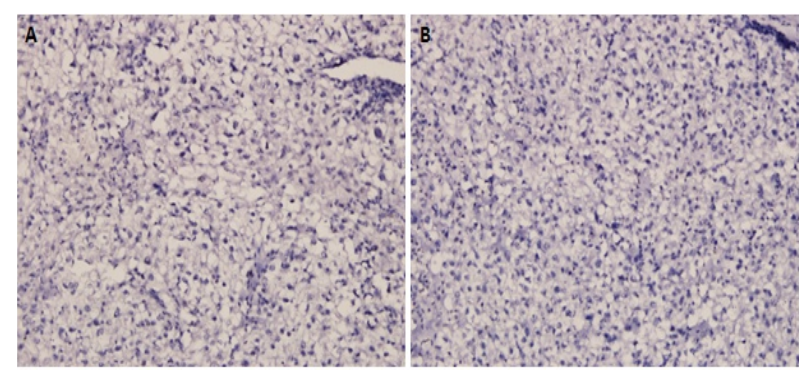

Figure 3. Negative Nuclear Staining of p53 and MDM2 in Renal Cell Carcinoma. (A) p53 Negative Immunoreaction with Original Magnification $\times 20$, (B) MDM2 Negative Immunoreaction with Original Magnification $\times 20$ this regard, 5-year mortality was $30.5 \%$ and thus 5-year survival was thus 5 -year survival was $85.3 \%$. There was no difference in 5-year survival between men and women $(86.4 \%$ versus $83.9 \%, \mathrm{p}=0.834)$. The mean age of non-survivors was significantly higher than survivors $(60.9 \pm 13.7$ years versus $55.0 \pm 11.4$ years, $p=0.039)$. Also, 5-year survival in clear type was Also, 5-year survival in clear type was $82 \%$, in chromophobe type was $100 \%$, and in papillary type was $77.8 \%(\mathrm{p}=0.014)$. 5-year survival rate was adversely associated with tumor grade (grade 1: $90 \%$, grade $2: 88.9 \%$, grade 3: $76.5 \%$, grade $4: 66.7 \%$, $\mathrm{p}=0.001$ ) and stage stage (stage I: $96.7 \%$, stage II: $90.9 \%$, stage III: $68.2 \%$, stage IV: $0 \%, \mathrm{p}=0.001)$. The higher intensity of immunostaining for anti-P53 antibody was

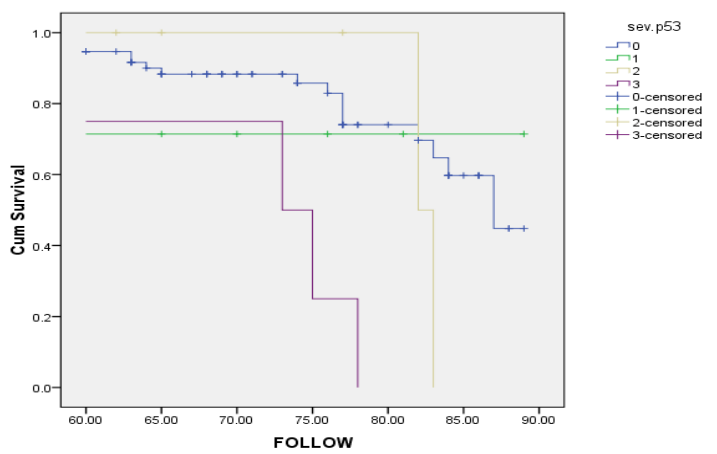

Figure 4. Association of Intensity of Immunostaining for Anti-p53 Antibody with Survival

Table 1. Association of p53 Intensity with Survival

\begin{tabular}{lcccc}
\hline Intensity of p53 & 0 & 1 & 2 & 3 \\
\hline Survival & & & & \\
5-years & $87.10 \%$ & $80.10 \%$ & $75 \%$ & $60 \%$ \\
6-years & $88.30 \%$ & $71.40 \%$ & $50 \%$ & $75 \%$ \\
7-years & $59.70 \%$ & $71.40 \%$ & $0 \%$ & $0 \%$ \\
\hline
\end{tabular}

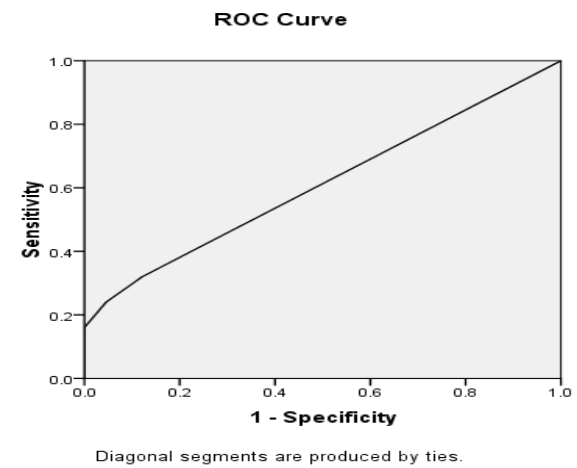

Area Under the Curve

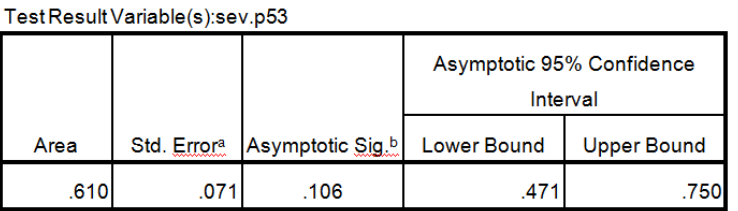

The test result variable(s): sev.p53 has at least one tie between the positive actual state group and the negative actual state group. Statistics may be biased. a. Under the nonparametric assumption

b. Null hypothesis: true area $=0.5$

Figure 5. ROC Curve for p53 Showing Limited Role for Predicting Long-term Survival 
Table 2. Association of MDM2 Intensity with Survival

\begin{tabular}{lcccc}
\hline MDM2 intensity & 0 & 1 & 2 & 3 \\
\hline Survival & & & & \\
5-years & $78.30 \%$ & $76.90 \%$ & $100.00 \%$ & $87.00 \%$ \\
6-years & $81.10 \%$ & $80.20 \%$ & $90.90 \%$ & $88.60 \%$ \\
7-years & $38.90 \%$ & $50.90 \%$ & $58.40 \%$ & $63.20 \%$ \\
\hline
\end{tabular}

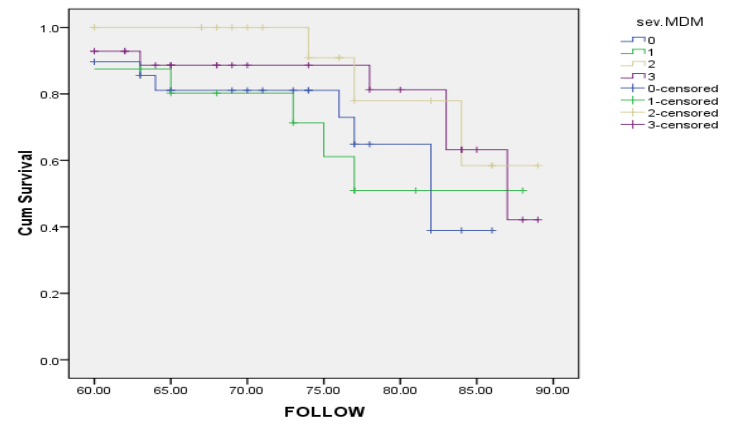

Figure 6. Association of Immunostaining for AntiMDM2 Antibody with Survival

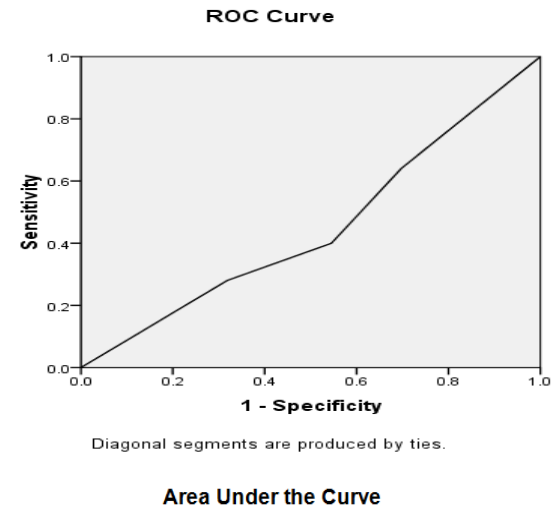

Test Result Variable(s):sev.MDM

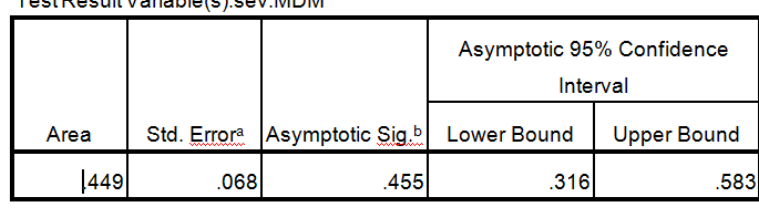

The test result variable(s): sev.MDM has at least one tie between the positive actual state group and the negative actual state group. Statistics may be biased.

a. Under the nonparametric assumption

b. Null hypothesis: true area $=05$

Figure 6. ROC Curve for MDM2 Showing MDM2 Positivity Could not Predict Survival

associated with lower 5-year survival $(87.1 \%$ for score $0,80.1 \%$ for score $1,75.0 \%$ for score 2 , and $60.0 \%$ for score 3, $\mathrm{p}=0.008$ ) (Figure 4) (Table 1), but no difference was found in 5-year survival between different scores for immunostaining for anti-MDM2 antibody $(78.3 \%$ for score $0,76.9 \%$ for score $1,100 \%$ for score 2 , and $87 \%$ for score 3, $\mathrm{p}=0.545$ ) (Figure 5) (Table 2). According to the Cox proportional hazard analysis, positive P53 marker was only predictor for patients' 5-year survival that the presence of positive p53 increased the risk for long-term mortality up to 2.8 times $(\mathrm{HR}=2.798,95 \% \mathrm{CI}$ : 1.176-6.660, $\mathrm{P}=0.020)$. However, the presence of MDM2 could not predict long-term mortality. In this regard, analysis by the ROC curve (figure 6) showed a limited role for predicting long-term survival by confirming p53 positivity (AUC=0.610, 95\%CI: 0.471-.750, $\mathrm{P}=0.106$ ). In this regard, the best cutoff point for P53 to predict mortality was 0.5 yielding a low sensitivity (32.0\%) but a high specificity (97.9\%). In similar analysis (figure 7), measurement of MDM2 positivity could not predict mortality (AUC=0.449, 95\%CI: 0.316-.583, $\mathrm{P}=0.455)$.

\section{Discussion}

The present study first aimed to determine the frequency of positive anti-P53 and also anti-MDM2 antibodies in patients who underwent radical nephrectomy leading a considerably low positivity of anti-P53 in $1.1 \%$, but a positivity of anti-MDM2 in one-fifth of study subjects. However, only $1.1 \%$ of all subjects had positivity for both markers. Second, we showed that both indices could not predict long-term mortality and thus long-term survival in the patients. In this regard, analyzing area under the ROC curve was not resulted in a proper cutoff value of both P53 and MDM2 for predicting 5-year survival in the patients. On the other hand, the present scoring system for both biomarkers regarding intensity and percentage of the cells staining seems to be not useful for assessing long-term mortality in our population. In this regard, we did not achieve homogeneity of findings in Cox modeling and ROC curve analysis.

Reviewing the literature reveals contradictory results. In a study (Hejnold et al., 2014), about $6.6 \%$ of patients were $\mathrm{P} 53+/ \mathrm{MDM} 2+$ that was higher compared to our results as $1.1 \%$. In another study (Noon et al., 2012), the presence of P53 but not its mutation was associated with reduced survival. Also, MDM2 expression was related to reduce survival. Other study (Polanski, 2010) showed that the concurrent expression of both p53 and MDM2 was associated with considerably decreasing survival. In another research (Uchida et al., 2002) the expression of P53 and MDM2 was showed in $13.4 \%$ and $1.8 \%$ that was contrary to our finding. In that study, the expression of both markers could predict poor outcome in the patients. In other study (Haitel et al., 2000) study, simultaneous expression of both P53 and MDM2 was related to tumor progression. There is also a similar (Hashimoto et al., 2000) that indicated that P53 could not predict disease outcome and thus only type of infiltration was predictor for poor outcome. Other research (Moch et al., 2013) also indicated positive $\mathrm{P} 53$ in $16 \%$ and positive MDM2 in 30\% of subjects with a strong association between the two biomarkers and also with poor prognosis of the disease. In total, it seems that both P53 and MDM2 markers in Iranian population may not be able to predict long-term survival in patients who underwent radial nephrectomy that can be affected by genetic and racial differences. However, this claim should be assessed in further studies.

In total, the simultaneous presence of both P53 and MDM2 markers in our population is a rare phenomenon and the presence of these markers may not predict long-term survival in patients who undergoing radical nephrectomy. Future multicenter studies with larger sample size are required to show the prevalence of P53 and MDM2 markers in Iranian population and association with long- 
term survival.

\section{Acknowledgements}

This study was supported by the Oncopathology Research Center, Iran University of Medical Sciences (Research No: 92-01-12-21798). The authors have no financial involvement with any organization or entity with a financial interest in or financial conflict with the subject matter or materials discussed in the manuscript apart from those disclosed. No writing assistance was utilized in the production of this manuscript.

\section{References}

Akbari ME, Hosseini SJ, Rezaee A, et al (2008). Incidence of genitourinary cancers in the Islamic Republic of Iran: a survey in 2005. Asian Pac J Cancer Prev, 9, 549-52.

Basiri A, Shakhssalim N, Jalaly NY, et al (2014). Difference in the incidences of the most prevalent urologic cancers from 2003 to 2009 in Iran. Asian Pac J Cancer Prev, 15, 1459-63.

Chow WH, Gridley G, Fraumeni JF Jr, Jarvholm B (2000). Obesity, hypertension, and the risk of kidney cancer in men. N Engl J Med, 343, 1305-11.

Chow WH, Dong LM, Devesa SS (2010). Epidemiology and risk factors for kidney cancer. Nat Rev Urol, 7, 245-57.

Haas NB, Nathanson KL (2014). Hereditary kidney cancer syndromes. Adv Chronic Kidney Dis, 21, 81-90.

Haitel A, Wiener HG, Baethge U, Marberger M, Susani M. $\mathrm{mdm} 2$ expression as a prognostic indicator in clear cell renal cell carcinoma: comparison with p53 overexpression and clinicopathological parameters. Clin Cancer Res, 6, 1840-4.

Hashimoto H, Sue Y, Saga Y, Tokumitsu M, Yachiku S (2000). Roles of p53 and MDM2 in tumor proliferation and determination of the prognosis of transitional cell carcinoma of the renal pelvis and ureter. Int J Urol, 7, 457-63.

Hejnold M, Dyduch G, Białas M, et al (2014). Selected immunohistochemical features of conventional renal cell carcinomas coexpressing P53 and MDM2. Pol J Pathol, 65, 113-9.

Jemal A, Murray T, Ward E, et al (2005). Cancer statistics, 2005. CA Cancer J Clin, 55, 10-30.

Karami S, Daugherty SE, Schonfeld SJ, et al (2013). Purdue MP Reproductive factors and kidney cancer risk in 2 US cohort studies, 1993-2010. Am J Epidemiol, 177, 1368-77.

Kim HL, Seligson D, Liu X, et al (2004). Using protein expressions to predict survival in clear cell renal carcinoma. Clin Cancer Res, 10, 5464-71.

Ljungberg B, Bozoky B, Kovacs G, et al (2001). p53 expression in correlation to clinical outcome in patients with renal cell carcinoma. Scand J Urol Nephrol, 35, 15-20.

Moch H (2013). An overview of renal cell cancer: pathology and genetics. Semin Cancer Bio, 23, 3-9.

Moore LE, Wilson RT, Campleman SL (2005). Lifestyle factors, exposures, genetic susceptibility, and renal cell cancer risk: a review. Cancer Invest. 23, 240-55.

Noon AP, Polański R, El-Fert AY, et al (2012). Combined p53 and MDM2 biomarker analysis shows a unique pattern of expression associated with poor prognosis in patients with renal cell carcinoma undergoing radical nephrectomy. $B J U$ Int, 109, 1250-7.

Olumi AF, Weidner N, Presti JC (2001). p53 immunoreactivity correlates with Ki-67 and bcl-2 expression in renal cell carcinoma. Urol Oncol, 6, 63-67.

Polanski BR (2010). Use of MDM2 to link survival and invasiveness in renal cell carcinoma and as a novel drug target for therapy. 2010 Genitourinary Cancers Symposium, Abstract No 320.

Sanfilippo KM, McTigue KM, Fidler CJ, et al (2014). Hypertension and obesity and the risk of kidney cancer in 2 large cohorts of US men and women. Hypertension, 63, 934-41.

Shuch B, Vourganti S, Ricketts CJ, et al (2014). Defining earlyonset kidney cancer: implications for germline and somatic mutation testing and clinical management. J ClinOncol, 32, 431-7.

Uchida T, Gao JP, Wang C, et al (2002). Clinical significance of $\mathrm{p} 53, \mathrm{mdm} 2$, and bcl-2 proteins in renal cell carcinoma. Urology, 59, 615-20.

Uzunlar AK, Sahin H, Yilmaz F, Ozekinci S (2005). Expression of $\mathrm{p} 53$ oncoprotein and bcl-2 in renal cell carcinoma. Saudi Med J, 26, 37-41.

Washio M, Mori M, Mikami K, et al (2014). Risk factors for renal cell carcinoma in a Japanese population. Asian Pac J Cancer Prev, 15, 9065-70.

Yap NY, Ng KL, Ong TA, et al (2013), Clinical prognostic factors and survival outcome in renal cell carcinoma patients--a malaysian single centre perspective. Asian Pac J Cancer Prev, 14, 7497-500.

Zigeuner R, Ratschek M, Rehak P, Schips L, Langner C (2004). Value of p53 as a prognostic marker in histologic subtypes of renal cell carcinoma: a systematic analysis of primary and metastatic tumor tissue. Urology, 63, 651-5. 